\section{SP1-74 THE PREVALENCE OF IRON DEFICIENCY ANAEMIA AND A COMPARISON OF THE INTAKE OF IRON AMONG PREGNANT WOMEN WITH THE DIETARY REFERENCE INTAKES FOR JAPANESE}

doi:10.1136/jech.2011.142976n.51

\begin{abstract}
${ }^{1,2} \mathrm{M}$ Sawada, ${ }^{1} \mathrm{Y}$ Kobayashi, ${ }^{1} \mathrm{Y}$ Tanaka, ${ }^{1,3} \mathrm{C}$ Shigemura, ${ }^{1} \mathrm{~K}$ Harada, ${ }^{4} \mathrm{H}$ Tamura, ${ }^{4} \mathrm{H}$ Tamura, ${ }^{5} \mathrm{M}$ Matsumoto, ${ }^{6} \mathrm{H}$ Asano, ${ }^{7} \mathrm{~N}$ Hagiwara, ${ }^{8} \mathrm{I}$ Kitagawa, ${ }^{9} \mathrm{~J} \mathrm{Ikeda,}{ }^{1} \mathrm{Y}$ Kido, ${ }^{1}$ A Higashi. ${ }^{1}$ Graduate School of Life and Environmental Sciences, Kyoto Prefectural University, Kyoto, Japan; ${ }^{2}$ Kyoto Patisserie Art College, Kyoto, Japan; ${ }^{3}$ Kyoto College of Nutritional \& Medical Sciences, Kyoto, Japan; ${ }^{4}$ Tamura Ladies Clinic, Kameoka, Japan; ${ }^{5}$ Department of Nutritional Management, University Hospital, Kyoto Prefectural University of Medicine, Kyoto, Japan; ${ }^{6}$ School of Nursing, Kyoto Prefectural University of Medicine, Kyoto, Japan; ${ }^{7}$ Faculty of Home Sciences and Welfare, Kyoto Notre Dame University, Kyoto, Japan; ${ }^{8}$ Faculty of Human Life and Environment, Nara Women's University, Nara, Japan; ${ }^{9}$ Kyoto Bunkyo Junior College, Uji, Japan
\end{abstract}

Introduction In order to determine the prevalence of anaemia and to explore the associations between iron status and intake in the process of pregnancy, we compared the dietary and total intake of iron with the Dietary Reference Intakes (DRIs) for Japanese in a population of pregnant women in the suburbs.

Methods A total of 109 pregnant women participated in the survey and were measured for blood haemoglobin $(\mathrm{Hb})$. For 42 (mean age \pm SD: $30.2 \pm 4.5$ ) clinically normal pregnant women (Hb concentration $>11 \mathrm{~g} / \mathrm{dl}$ ) in the first trimester of pregnancy, we used the dietary record methods for two consecutive weekdays and a weekend day with a handy camera, to examine dietary intake of iron in the second trimester. Dietary intake analysis was performed using Healthy Maker Pro 501 software, Mushroom-soft. Statistical analysis was performed with SPSS for Windows, Version 11.5. Blood haemoglobin, haematocrit, serum iron, and ferritin were measured at the third trimester.

Results At the first trimester, anaemia was diagnosed in $48.6 \%$ of the subjects $(\mathrm{Hb}<11.0 \mathrm{~g} / \mathrm{dl})$. At the second trimester, iron intake was lower than the estimated average requirement of DRIs $(16.5 \mathrm{mg} /$ day $)$ in $93 \%$ of the subjects. The level of latent iron deficiency anaemia (Ferritin $<12 \mathrm{ng} / \mathrm{dl}$ ) was $88.1 \%$ and the anaemia $(\mathrm{Hb}$ $<11.0 \mathrm{~g} / \mathrm{dl}$ ) was $52.4 \%$ at the third trimester.

Conclusion The results of our study support that the iron deficiency anaemia is a physiological adaptation for prevention of thrombosis during pregnancy.

\section{SP1-75 MULTIPLE IMPUTATION AND SURVIVAL ANALYSIS: AN EXAMPLE USING CANCER REGISTRY DATA}

doi:10.1136/jech.2011.142976n.52

${ }^{1} \mathrm{M}$ van Laar, * ${ }^{2} \mathrm{D}$ P Stark, ${ }^{1} \mathrm{R}$ G Feltbower. ${ }^{1}$ Paediatric Epidemiology Group, University of Leeds, Leeds, UK; ${ }^{2}$ Institute of Oncology, Leeds Teaching Hospitals NHS Trust, Leeds, UK

Introduction Stage of disease at presentation of cancer is not routinely recorded in medical records, resulting in large amounts of missing data. We investigated survival trends by ethnicity among 0-29 year olds with cancer while using multiple imputation (MI) to impute missing values of stage.

Method Subjects $(n=3534)$ diagnosed with cancer in Yorkshire (1990-2005) were analysed. Individual imputation models were used to assign stage of disease to four main diagnostic groups; leukaemia, lymphoma, central nervous system (CNS) and other solid tumours. Linear regression was used to impute white blood cell count (WCC) for leukaemia as a proxy for stage and ordinal logistic regression was used to impute for the remaining diagnostic groups. The survival analysis was performed using Cox regression.
Results Missing stage data occurred in $66 \%$ of cases for lymphoma $28 \%$ for CNS tumours, and $69 \%$ for other solid tumours. WCC was missing for $57 \%$ of leukaemia cases. Results of the final analysis showed an increased risk of death for south Asians compared to non-south Asians with leukaemia (HR 1.61; 95\% CI 1.01 to 2.55) and lymphoma (HR 2.05; 95\% CI 1.09 to 3.87), and a decreased risk for south Asians with other solid tumours (HR 0.50; 95\% CI 0.28 to 0.89). There was no significant difference by ethnic group for those with CNS tumours (HR 1.51; 95\% CI 0.82 to 2.78).

Conclusion Although stage was missing in two-thirds of cases overall, MI was used to minimise bias and enhance the precision of analyses. This technique therefore offers considerable advantages over other approaches such as complete case analysis or coding missing data as a separate category.

\section{SP1-76 DIFFERENCES IN CARDIOVASCULAR RISK FACTORS BY MUNICIPALITY POPULATION SIZE: NATIONAL HEALTH AND NUTRITION SURVEY, JAPAN}

doi:10.1136/jech.2011.142976n.53

${ }^{1} \mathrm{~N}$ Nishi, ${ }^{*} \mathrm{M}$ Nozue, ${ }^{1} \mathrm{~N}$ Sarukura, ${ }^{1} \mathrm{M}$ Nakade, ${ }^{2} \mathrm{Y}$ Arai, ${ }^{3} \mathrm{~K}$ Yoshita, ${ }^{4} \mathrm{~N}$ Yoshiike ${ }^{1}$ National Institute of Health and Nutrition, Tokyo, Japan; ${ }^{2}$ Chiba Prefectural University of Health Sciences, Chiba, Japan; ${ }^{3}$ Osaka City University, Osaka, Japan; ${ }^{4}$ Aomori University of Health and Welfare, Aomori, Japan

Introduction The National Health and Nutrition Survey (NHNS) has been conducted for more than 60 years in Japan. A total of 300 survey districts are randomly selected from all over Japan, and they can be categorised by population size of municipalities to which they belong. This study aimed to compare cardiovascular risk factors by population size using the NHNS data.

Methods Subjects were 3311 men and 3843 women aged 30 years and over of the NHNS in 2006. Survey districts were categorised into three groups by municipality population size: 150000 and over (large), 50000-149999 (medium), and $<50000$ (small). As for cardiovascular risk factors, we used body mass index, waist circumference, systolic and diastolic blood pressure, total cholesterol, HDL cholesterol, HbA1c and current smoking. Age was adjusted using multilevel regression model where individuals at level 1 were nested within survey districts at level 2. Models were fitted by MLwiN 2.02. Results Compared with survey districts in large municipalities, $\mathrm{HbA1c}$ was lower in survey districts in medium municipalities and total and HDL-cholesterol were lower and the percentage of current smokers was higher in survey districts in small municipalities in men, and the percentage of current smokers was lower in survey districts in medium municipalities in women. These differences were statistically significant, but their magnitudes were rather small.

Conclusion No remarkable differences in cardiovascular risk factors were observed by municipality population size in Japan, but their trends should be monitored continually.

\section{SP1-77 STRATEGY FOR THE CARE OF THE ELDERLY WITH LIMITED FUNCTIONAL CAPACITY IN A DEVELOPING COUNTRY}

doi:10.1136/jech.2011.142976n.54

${ }^{1} V$ C Paduan, ${ }^{*}$ A T de Abreu Ramos-Cerqueira, ${ }^{2}$ C S R L Henri, ${ }^{2} \mathrm{~N}$ I L Oliveira, ${ }^{1} \mathrm{~J}$ C N Marchette. ${ }^{1}$ Faculdade de Medicina de Botucatu, Botucatu/São Paulo, Brazil, ${ }^{2}$ Centro de Convivência do Idoso-Aconchego, Botucatu/São Paulo, Brazil

Background The "Aconchego" Day Center (ADC) aims to care for elderly individuals with limited functional capacity, an unprecedented strategy in a medium-sized city in the State of São Paulo (Brazil). It was created 10 years ago, due to the high degree of emotional burden of elderly caregivers and lack of community care resources for the elderly or support for the families. The ADC is a non- 
governmental organisation and offers free space for social interaction, cognitive and physical stimulation, guidance and support to caregivers.

Objective Describe the sociodemographic, cognitive and affective aspects of the elderly $(n=46)$ and their caregivers $(n=34)$ through a cross-sectional descriptive study evaluating cognitive functioning, dementia, depressive symptoms, performance in basic (BADLs) and instrumental (IADLs) activities of daily living, and emotional overload and common mental disorder of the caregiver.

Results The mean age of the elderly individuals was 77 years-old (SD \pm 9 ), $76 \%$ were women, $67 \%$ live without a partner, $93 \%$ were retired and $50 \%$ had $<4$ years schooling. Average participation in the ADC was 24 months, $79 \%$ presented cognitive impairment and $57 \%$ mild, moderate or severe dementia. For BADLs, $94 \%$ showed some dependence on caregivers, while for IADLs, 100\% were dependent. Among the elderly, $35 \%$ presented depressive symptoms that are correlated with the presence of cognitive impairment $(p=0.004)$. Among the caregivers, $47 \%$ reported emotional overload, while $41 \%$ presented common mental disorders. The relevance of the proposal was verified, considering the fragility of the elderly and the burden and emotional distress of caregivers.

\section{SP1-78 ASTHMA-RELATED HOSPITAL MORBIDITY IN POLAND}

doi:10.1136/jech.2011.142976n.55

${ }^{1} \mathrm{~A}$ Pac, ${ }^{*}{ }^{1} \mathrm{P}$ Gorynski. ${ }^{1}$ Jagiellonian University Medical College, Chair of Epidemiology and Preventive Medicine, Krakow, Poland; ${ }^{2}$ National Institute of Public Health, National Institute of Higiene, Warsaw, Poland

Introduction Asthma prevalence is growing in many countries. The prevalence of asthma is particularly high in developed countries. In Poland asthma morbidity is growing. The aim of this study was to assess the hospital morbidity in Poland and to examine the areabased factors area (socioeconomic, accessibility to the health services and air pollution) that influence morbidity.

Methods We used data from the "Hospitalisation" project in which the National Institute of Health, National Institute of Hygiene collect data on hospital admissions across the country. An asthma hospitalisation was defined as a hospitalisation ICD 10 coded J45 or J46 between 2004 and 2006. Data on the population, healthcare accessibility and the level of air pollution at the voivodship level for the same years was also used.

Results An increase in asthma hospitalisation rates was observed over the study period with regional variation in hospital morbidity. Asthma hospitalisation rates were related to the level of average wage $(\beta=-1.69, p<0.001)$, physician accessibility $(\beta=1.50, p=0.002)$, the percentage of young people in population $(\beta=-0.43, p=0.040)$ and sulphur dioxide air pollution level $(\beta=-0.79 ; p=0.006)$.

Conclusions There is high variation in asthma hospital morbidity according to voivodships in Poland which can be partially explained, at the population level, by access to healthcare services and air pollution.

\section{SP1-79 BIRTH ORDER, GESTATIONAL AGE AND BIRTH WEIGHT AND THE AGE AT ONSET OF PUBERTY: EVIDENCE FROM HONG KONG'S "CHILDREN OF 1997" BIRTH COHORT}

doi:10.1136/jech.2011.142976n.56

L L Hui, ${ }^{*}$ G M Leung, T H Lam, C M Schooling. Life course and Lifestyle Epidemiology Group, School of Public Health, Li Ka Shing Faculty of Medicine, The University of Hong Kong, Hong Kong SAR, China

Introduction Intrauterine and pubertal factors are associated with reproductive and cardiovascular health in both sexes. It is unclear how intrauterine exposures, birth order, gestational age and birth weight, also affect the timing of puberty.
Methods We used interval-censored survival analyses in 3750 boys and 3241 girls (84\% follow-up) in a Chinese birth cohort, "Children of 1997", comprising 88\% of births in Hong Kong in April and May 1997, to examine the adjusted associations of birth order, gestational age and birth weight with age at onset of puberty (Tanner stage II). We also examined whether the associations varied with sex or height at 7 years.

Results Birth order and birth weight were unrelated to the age at onset of puberty, adjusted for sex, gestational age and socioeconomic position. Gestational age had a sex-specific association with age at onset of puberty, and was associated with earlier onset among girls (Time Ratio $0.994,95 \%$ CI 0.991 to 0.997 ) but not boys. None of these associations varied with childhood height.

Conclusion Intrauterine exposures, as proxied by gestational age, birth order and birth weight, had little impact on the timing of the onset of puberty, which was only evident for gestational age among girls. Given that it is unclear whether onset, duration or intensity of puberty is more relevant to the detrimental consequences of early puberty, further studies investigating intrauterine, infant and childhood influences on the duration and intensity of puberty may help unravel the early origins of cardiovascular diseases and breast cancer.

\section{SP1-80 PROGRAM EVALUATION FAMILIAL CANCER GENETIC COUNSELLING IN THE COMMUNITY OF VALENCIA. SPAIN (2005-2010)}

doi:10.1136/jech.2011.142976n.57

1,2M G Sáez, * 1,2D S Trejo. ' Oficina del Plan del Cáncer (Dirección General de Salud Pública), Valencia, Spain; ${ }^{2}$ Centro Superior de Investigación en Salud Pública (CSISP), Valencia, Spain

Background In 2005 the Health Department of the Valencia (Spain), implemented a program of familial cancer. The objective of this program is to reduce cancer incidence and mortality in those with a known genetic predisposition. Through Genetic Counselling Units in Cancer (UCGC) a multidisciplinary team provides information, recommendations and follow-up more appropriate for the patient and family.

Method Descriptive analysis from the start of the program in May 2005 to December 2010, through indicators of organisation and effectiveness: number of people who come to the UCGC, average size of families, rates, appropriate referrals, families with identified mutations, and distribution of the gene results for index cases and family. Results 9.942 people have been treated, 870 was referred from primary care and 9.072 from specialty care. $79 \%$ of the consultations met criteria for genetic studies. The 59\% related to the syndrome of breast and ovarian cancer and $32 \%$ relative to the HNPCC syndrome. Some case index was identified from 3.083 families $(78.7 \%)$ and in $91.3 \%$ of them have done a genetic study. Pathogenic mutations were detected in $21.8 \%$ of these studies. Study is offered to families where pathogenic mutation has been found. The percentage of pathogenic mutations diagnosed in family is approximately $54 \%$.

Conclusions The diagnosis and genetic counselling in cancer, as part of an organised program intended to diagnose early-stage disease or prevent it. To make an assessment of the impact of this program on the health of the population would need to follow the very long term.

\section{SP1-81 AN APPROACH TO COMPARE THE IMPORTANCE OF DIFFERENT CARDIOVASCULAR EXPOSURES AS CONTINUOUS VARIABLES}

doi:10.1136/jech.2011.142976n.58

${ }^{1,2} \mathrm{D}$ Khalili, ${ }^{1} \mathrm{Y}$ Mehrabi, ${ }^{2} \mathrm{~F}$ Hadaegh, ${ }^{2} \mathrm{~N}$ Fahimfar, ${ }^{2} \mathrm{~F}$ Azizi. ${ }^{1}$ School of public health, Shahid Beheshti University of Medical Sciences, Tehran, Iran; ${ }^{2}$ Research 